109 A

The Carry-over Effects of Triazolam Compared with Nitrazepam and Placebo in Acute Emergency Driving Situations and in Monotonous Simulated Driving

Hans Laurell and Jan Törnros

Reprint from Acta Pharmacologica et toxicologica 1986 


\title{
The Carry-over Effects of Triazolam Compared with Nitrazepam and Placebo in Acute Emergency Driving Situations and in Monotonous Simulated Driving
}

\author{
By \\ Hans Laurell and Jan Törnros \\ (Received October 9, 1985; Accepted January 9, 1986)
}

\begin{abstract}
Eighteen healthy volunteers of both sexes, aged 20-34, were tested in the morning while undertaking real car driving avoidance manoeuvres and during monotonous simulated driving after 1 and 3 nights of medication with triazolam $0.25 \mathrm{mg}$, nitrazepam $5 \mathrm{mg}$ or placebo. The study was a double-blind, randomized, cross-over study, where a minimum of 7 days wash-out separated the 3 treatment periods. Nitrazepam was found to impair performance in the simulated task after 1 but not after 3 nights of medication. Performance in the triazolam condition was not significantly different from the other conditions on this task on either day. However, after one night of medication triazolam tended to score worse than placebo but better than nitrazepam. In real car driving a tendency was noted for nitrazepam to score worst, whereas the difference between placebo and triazolam was hardly noticeable. The same tendency appeared on both days.
\end{abstract}

Key-words: Driver performance - drug driving simulator.

In recent years several new benzodiazepine derivatives have been developed in search of hypnotics with effective hypnotic activity but which do not produce unwanted residual effects. This has great social implications especially in societies where transportation mainly relies upon private cars. A state of continual alertness is of critical importance when driving a car. The the wide use however, of sleeping pills may give rise to hang-over effects that can impair driving performance.

Triazolam is a triazolobenzodiazepine differing from most others in that it is active in very low doses and has a short mean elimination half-life of 2.3 hrs (Eberts et al. 1981). Thus, it would reduce the risk of residual effects.

In this study, a comparison is made between triazolam and nitrazepam (with a mean elimination half-life of $29 \mathrm{hrs}$ ) (Kangas et al. 1979) and placebo.
When comparing the possible residual effects of various hypnotics on car driving performance, two aspects of driving stand out as particularly interesting, namely monotonous driving and emergency avoidance situations. By employing such test situations, the study would be able to yield information that concerns performance, related to driving in heavy city traffic as well as on empty highways.

\section{Materials and Methods}

Eighteen healthy volunteers of both sexes, aged 20-34, who possessed a current Swedish Car Driving License, were enrolled in the study.

The trial employed a double-blind, randomized, crossover design. The study medications, triazolam $0.25 \mathrm{mg}$, nitrazepam $5 \mathrm{mg}$ and placebo were each administered for 3 consecutive nights with a minimum of 7 days 
between each treatment period. Assessments were made in the morning after 1 and 3 nights of medication.

Simulated driving task. In order to detect any changes in susceptibility to driver fatigue, a monotonous $2.5 \mathrm{hrs}$ driving task was created in a driving simulator. The driving compartment of the simulator consisted of a real, truncated car body, complete with all controls, which were parts of a feed-back system of which a digitalanalogue computer was the brain.

A continually changing road pattern and landscape was randomly generated by a computer. A large television unit interpreted the signals and presented them visually on a wide screen in front of the driver. The programme allowed for an everchanging pattern of road and landscape which included not only bends and straights but also up and down-hill sections. Technological adaptions further mimicked real driving conditions by simulating vibrations on the road surface which, should the driver accidentally stray off the road onto the verge, increased in intensity.

Activation of the controls by the driver caused the appropriate change in the visual scenario to occur i.e. turning, braking, etc. The driver was instructed to stay on the right side of the road and to maintain a steady $90 \mathrm{~km} / \mathrm{hr}$ throughout the test.

The computer continually monitored the driver's performance and functioning and at the termination of a test run presented a detailed assessment of the parameters under study, brake reaction time, time off road or "across white line" etc.

Whilst driving the simulator the subject was exposed to a number of stimuli which, once perceived, called for a specific action to be taken. These events were preprogrammed and there was no way that the driver could predict their presentation in advance.

1. Visual signals. Lights were placed both in the central visual fields and to the periphery (see fig. 1). When they were illuminated the driver had to apply the brake immediately. The time from stimulus appearance to application of the brake was the "BRAKE REACTION TIME".

2. Auditory signals. An auditory tone, called for the same reaction as the visual stimuli already described.

Both the visual and auditory stimuli were presented randomly at intervals of between $10-120 \mathrm{sec}$.

Real car driving. In the real car driving situation, in contrast to the simulated monotonous task an emergency, evasive manoeuvre, was used (see fig. 2).

The driving task was largely the same as the one used in earlier studies on the effects of alcohol on driving performance (Laurell 1977; Laurell \& Törnros 1983). On observing an avoidance signal from an apparatus mounted on the front of the vehicle, the driver had to carry out an avoidance manoeuvre and, in doing this, try to avoid knocking over pylon cones which were placed along the avoidance path. The tolerance on either side of the car was approximately $15 \mathrm{~cm}$. The number of cones knocked over was employed as a measure of driver performance. The signal could be presented at either one of four positions in the cone setting. If presented above the left headlight position, the signal appeared as an obstacle in front of and to the left of the car, thus requiring an avoidance manoeuvre to the right, and vice versa.

The order of presentation of positions was randomized for each subject and each treatment condition. In each session, the course was negotiated 10 times plus 2 blank runs by each subject. Two warm-up trials identical to a test trial preceded each session. The total of 14 trials lasted $30 \mathrm{~min}$.

The road surface conditions are liable to considerable variations so the test area was sprinkled with water at regular intervals to maintain constant driving conditions. Vehicle speed was controlled by an automatic speed control system.

In order to keep motivation at a high level throughout the investigation, the amount of payment was made dependent upon performance. For each trial the driver

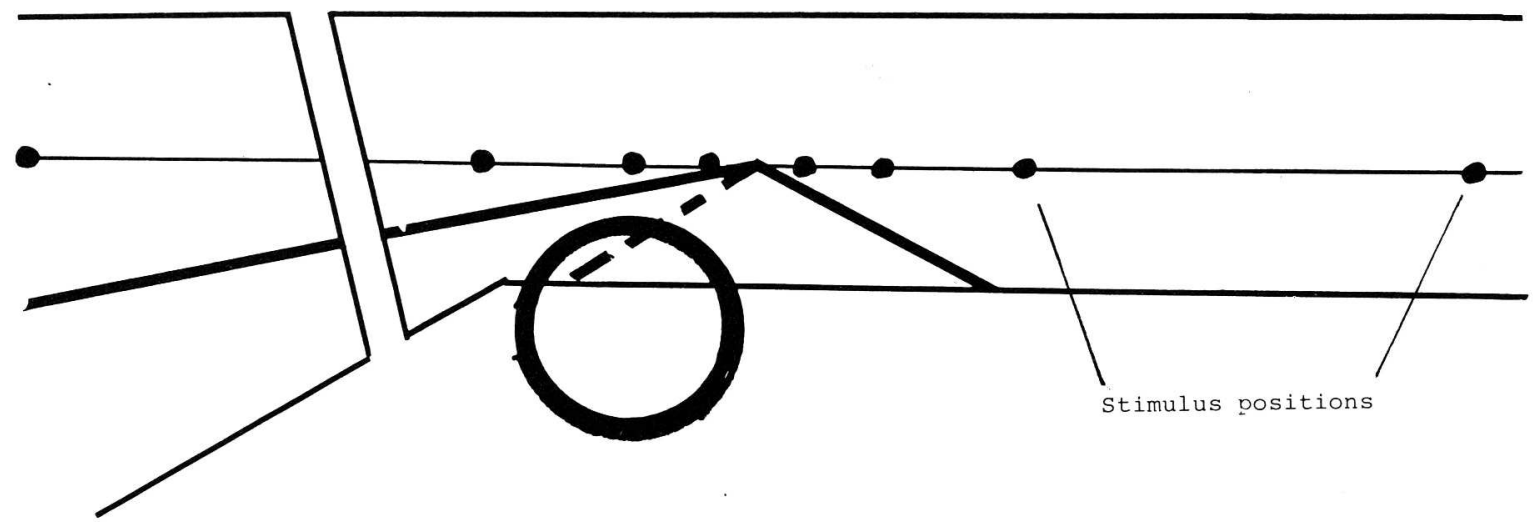

Fig. 1. Stimulus presentation in the driving simulator. Driver's view. 


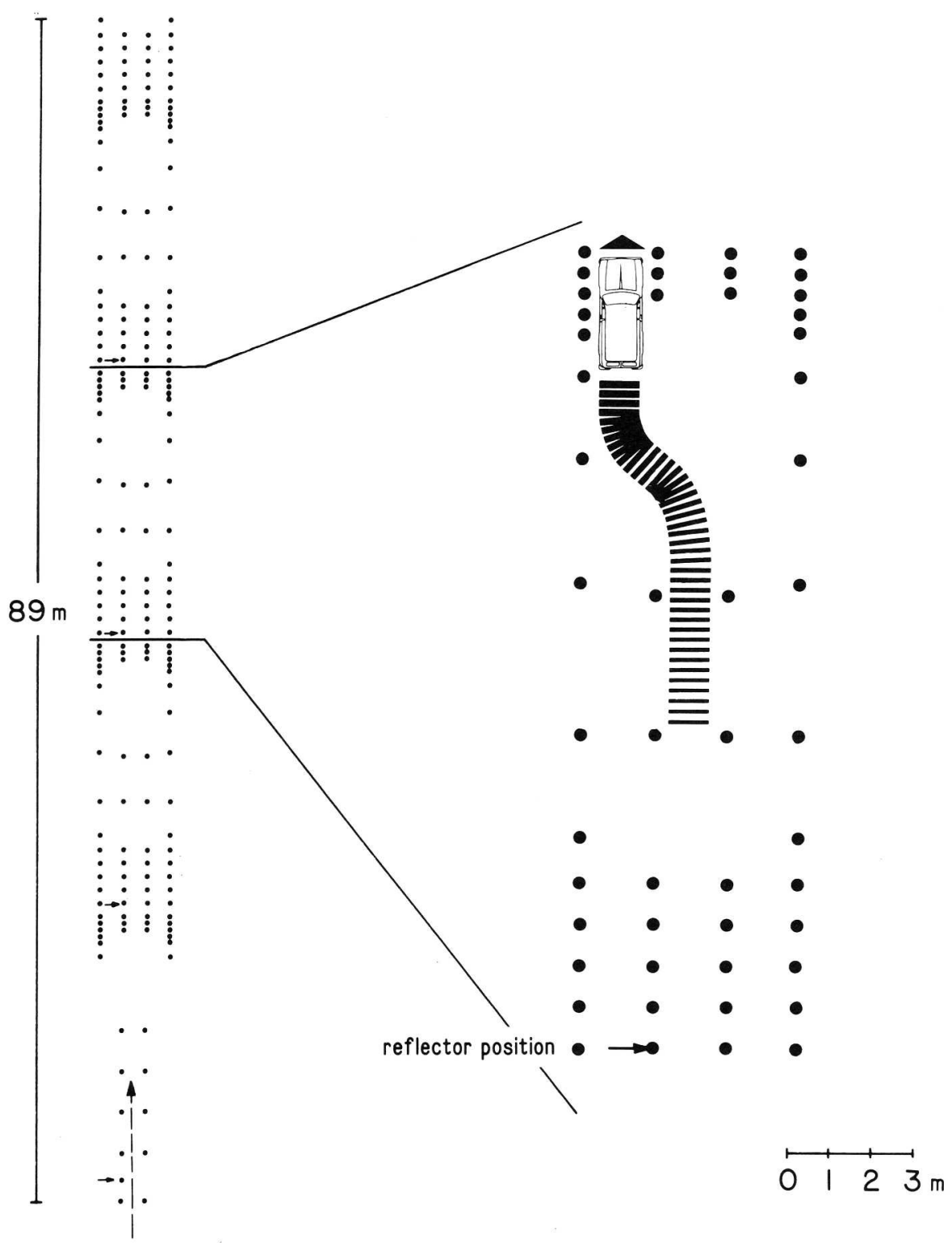

Fig. 2. Specifications of pylon arrangement.

had at his disposal a sum of 25 SEK (approximately 3.3 US\$). The sum of money was reduced for each cone knocked over. In the simulated monotonous driving task long reaction times $(>1.5 \mathrm{sec}$.) caused minor financial penalties.

Procedure. All subjects practiced the simulator task for one hour and the real car driving task for at least 2.5 hrs or until a minimum level of performance had been reached.

The capsules were taken at 11 p.m. after which the subjects went to bed. After a standardized breakfast in the morning, they were brought to the simulator for testing at $8 \mathrm{a} . \mathrm{m}$. Immediately after having completed the simulator test they took on the actual car driving task which lasted for $30 \mathrm{~min}$. Two days later the whole pro- cedure was repeated to examine the effects of possible drug accumulation.

\section{Results}

\section{Real car driving.}

Although the differences did not achieve statistical significance, (drug effect: $F(2,34)=2.05 ;$ n.s. Drug $\times$ day interaction $=\mathrm{F}(2,34)<1$; n.s.) the worst performance on both assessment days 1 and 3 was found with nitrazepam, whilst triazolam and placebo appeared to have about the same effect (fig. 3). 

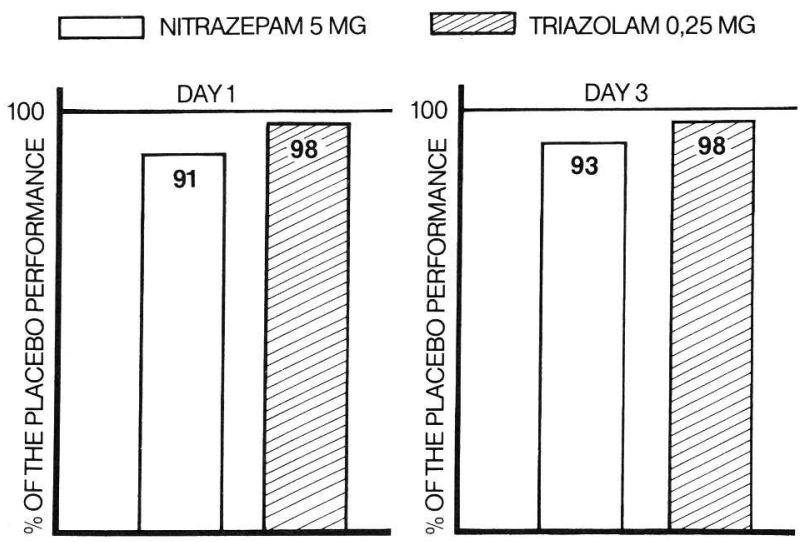

Fig. 3. Actual car driving (total means, 18 subjects).

\section{Monotonous simulated driving task.}

The data to be analyzed were the following: for every $10 \mathrm{~min}$. period of each test condition four mean reaction times were calculated for each subject, one for each stimulus mode/position: auditory, central-visual (4 positions), near peripheralvisual ( 2 positions) and far peripheral-visual (2 positions). Missed signals were omitted and were analyzed separately.

The trend noted in the real car driving was also found in the simulated condition, but only on day 1 , where the difference between drug conditions was significant $(\mathrm{F}(2,34)=3.45 ; \mathrm{P}<0.05)$. Closer examination revealed that the difference between nitrazepam and placebo was significant (Tukey's test: $\mathrm{q}=3.69$; $\mathrm{P}<0.05$ ), but the other two differences were not [nitrazepam-triazolam: $q=2.39$; $\mathrm{P}>0.05$ and triazolam-placebo: $\mathrm{q}=1.30 ; \mathrm{P}>0.05$ (Tukey's test)].

Drug treatment on day 1 did not interact in a significant way with either time on task $(\mathrm{F}(28,476)<1)$ or stimulus mode/position $[\mathrm{F}(6,102)=2.00 ; \mathrm{P}>0.05]$. Nor was the three-factor interaction significant ( $\mathrm{drug} \times$ time on task $\times$ stimulus mode/position: $\mathrm{F}(84,1428)=1.04 ; \mathrm{P}>$ $0.05)$. On day 3 no effect of drug treatment could be noticed, neither alone nor in combination with the other two factors (Drug: $\mathrm{F}(2,34)<1$. Drug $\times$ time on task: $F(28,476)=1.03 ; \mathrm{P}>0.05$. Drug $\times$ stimulus mode/position: $\mathrm{F}(6,102)<1$. Drug $\times$ time on task $\times$ stimulus mode/position: $\mathrm{F}(84,1428)=1.04 ; \mathrm{P}>0.05)$.

Fig. 4 shows the nature of the results regarding reaction time performance in the simulated task.

Very few signals were missed, a total of 29 (out of 8100). The nitrazepam condition, however, scored worst on both days (10 and 6 missed signals, respectively). The triazolam condition scored best on both days ( 2 on both days) with the placebo condition scoring in between ( 5 and 4 missed signals respectively). Very few subjects caused these misses and it was not considered meaningful to test these differences with respect to statistical significance.

\section{Discussion}

With the small doses of the test drugs administered only small effects of the treatments on performance were detected.

An increase in the Brake Reaction Time has been proven to reflect a decrease in the driving performance, particularly in the ability of the subject to detect obstacles along the road (Laurell \& Lisper 1978).

Examination of this dependent variable in our study showed a difference in level after one night of medication; reaction time was significantly longer in the nitrazepam condition than in the placebo condition, with the triazolam condition falling in between. No significant interaction between drug treatment and any of the other two factors (time on task, stimulus mode/position) appeared, however.

After 3 nights of medication there were few

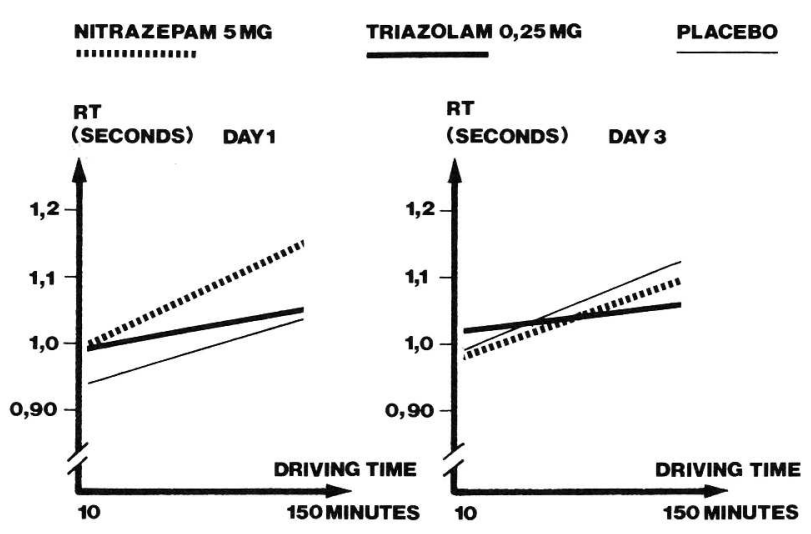

Fig. 4. Brake reaction times, regressions (total means, 18 subjects). The inclination of the curve shows how driving time in the simulator affects brake reaction times. 
differences favouring one treatment over the other, and there was no accentuation of the performance degradation noted following nitrazepam after the first night's medication. In fact no drug condition scored significantly worse than any other. It is not inconceivable that an adaptation to the presence of the drug might be at least partly responsible for this result.

On the Real Car Driving Task, no statistically significant differences in performance were found. Nitrazepam, however, tended to score worst whilst the difference between the placebo and triazolam conditions was hardly noticeable.

In conclusion it can be stated that the effect of drug treatment was small and appeared significant only in the simulated monotonous driving task after one night of medication. If the results regarding missed signals are included in the analyses, the effect is strengthened. However, a word of caution is required. The degrading effect of nitrazepam after one night of medication was certainly expected. Even so, this result can hardly be regarded as a scientific fact that needs no further supporting data. Considering the number of significance tests performed at the rather low level of significance $(\mathrm{P}<0.05)$ one has to be aware of the fact that the risk for the one significant effect found to be a spurious significance is not negligible.

The difference in performance between nitrazepam and triazolam after one night of medication was almost as great as that between nitra- zepam and placebo, although not statistically significant with the numbers of subjects studied. It should be noted, furthermore, that the degrading effects of the low dose of nitrazepam used were small on carry-over performance. It is likely that if higher doses of the drugs had been administered, this underlying trend would have been accentuated and probably led to larger and presumably significant differences in favour of the drug with the short half-life (Hindmarch \& Clyde 1980).

\section{References}

Eberts, F. S., Y. Philopoulus Jr., L. M. Reineke \& R. W. Vliek: Triazolam disposition. Clin. Pharmacol. Therap. 1981, 29, 81-93.

Hindmarch, J. \& C. A. Clyde: The effects of triazolam and psychomotor performance. Arzneimittelforsch. 1980, 30, 1163-1166.

Kangas, L., E. Iisalo, J. Kanto, V. Lehtinen, S. Pynnönen, I. Ruikka, J. Salminen, M. Sillanpää \& E. Syvälahti: Human pharmacokinetics of nitrazepam: Effect of age and diseases. Eur. J. Clin. Pharmacol. 1979, 15, 163-170.

Laurell, H.: Effects of small doses of alcohol on driver performance in emergency driving situations. Accident. Anal. Prev. 1977, 9, 191-201.

Laurell, H. \& H.-O. Lisper: A validation of subsidiary reaction time against detection of road side obstacles during prolonged driving. Ergonomics 1978, 21-2, 81-88.

Laurell, H. \& J. Törnros: Investigation of alcoholic hang-over effects on driving performance. Blutalkohol 1983, 20, 489-499. 
$3 !$
$!$
3,

" 